\title{
Impacto do isolamento social ocasionado pela pandemia COVID- 19 sobre a saúde mental da população em geral: Uma revisão integrativa
}

\author{
Impact of social isolation caused by the COVID-19 pandemic on the mental health of the general
} population: An integrative review

Impacto del aislamiento social causado por la pandemia COVID-19 en la salud mental de la población en general: Una revisión integradora

Priscila Carvalho Fogaça

ORCID: https://orcid.org/0000-0003-2787-2442 Universidade Luterana do Brasil, Brasil

E-mail: priscila.fogaca@ulbra.br

Guilherme Anzilero Arossi

ORCID: https://orcid.org/0000-0001-9580-6282 Universidade Luterana do Brasil, Brasil

E-mail: guilherme.arossi@ulbra.br

Alice Hirdes

ORCID: https://orcid.org/0000-0003-3318-0514 Universidade Luterana do Brasil, Brasil

E-mail: alice.hirdes@ulbra.br

\begin{abstract}
Resumo
Objetivo: investigar o impacto da pandemia causada pela COVID-19 sobre a saúde mental da população em geral. Método: trata-se de uma revisão integrativa da literatura, realizada através da busca nas bases de dados $P u b M e d$, Web of Science, PsycINFO, Scopus, BVS e Scielo, utilizando os descritores "SARS-CoV-2"; "COVID-19"; "Coronavirus"; "Mental health"; "Population". Foram identificados 1538 artigos, dos quais 16 foram incluídos neste estudo, sendo considerados elegíveis artigos originais e publicados em periódicos nacionais e internacionais, com data de publicação em 2020, disponíveis em língua portuguesa, inglesa e espanhola. Resultados: As evidências encontradas na literatura demonstram que indivíduos em isolamento social estão mais suscetíveis ao estresse e como consequência a esta privação social, tem apresentado principalmente transtornos de ansiedade, transtornos depressivos e a alteração na qualidade do sono. Foram observados também sentimentos de raiva, confusão e sintomas de estresse pós-traumático, que podem estar associados ao período de afastamento social prolongado, ao medo da contaminação, à frustração, à falta de suprimentos básicos, às informações insuficientes e a problemas financeiros. Conclusão: o enfrentamento da COVID-19 gerou uma mudança no modo de viver da sociedade, sendo necessário preparar os serviços em saúde para esta demanda, para que haja acolhimento e suporte psicológico e social da população geral, direcionando a atenção aos fatores, que desencadeiam o sofrimento psíquico, amparando os indivíduos neste período e nos pós- pandemia.
\end{abstract}

Palavras-chave: SARS-CoV-2; COVID-19; Coronavirus; Saúde mental; População.

\begin{abstract}
Objective: to investigate the impact of the pandemic caused by COVID-19 on the mental health of the general population. Method: this is an integrative literature review, carried out by searching the PubMed, Web of Science, PsycINFO, Scopus, BVS and Scielo databases, using the descriptors "SARS-CoV-2"; "COVID-19"; "Coronavirus"; "Mental health"; "Population". 1538 articles were identified, of which 16 were included in this study. Results: The evidence found in the literature shows that individuals in social isolation are more susceptible to the stress caused by this condition and as a consequence of this social deprivation has mainly presented anxiety disorders, depressive disorders and changes in sleep quality. Feelings of anger, confusion and symptoms of post-traumatic stress were also observed, which may be associated with the period of prolonged social withdrawal, fear of contamination, frustration, lack of basic supplies, insufficient information and financial problems. Conclusion: coping with COVID-19 generated a change in society's way of life, making it necessary to prepare health services for this demand, so that there is psychological and social support and support from the general population, directing attention to the factors that trigger suffering psychic, supporting individuals in this period and in the post-pandemic.
\end{abstract}

Keywords: SARS-CoV-2; COVID-19; Coronavirus; Mental health; Population. 


\begin{abstract}
Resumen
Objetivo: investigar el impacto de la pandemia causada por COVID-19 en la salud mental de la población en general. Método: se trata de una revisión integradora de la literatura, realizada mediante la búsqueda en las bases de datos PubMed, Web of Science, PsycINFO, Scopus, BVS y Scielo, utilizando los descriptores "SARS-CoV-2"; "COVID19"; "Coronavirus"; "Salud mental"; "Población". Se identificaron 1538 artículos, de los cuales 16 fueron incluidos en este estudio. Resultados: La evidencia encontrada en la literatura muestra que los individuos en aislamiento social son más susceptibles al estrés que genera esta condición y como consecuencia de esta privación social, ha presentado principalmente trastornos de ansiedad, trastornos depresivos y alteraciones en la calidad del sueño. También se observaron sentimientos de enfado, confusión y síntomas de estrés postraumático, que pueden estar asociados al período de retraimiento social prolongado, miedo a la contaminación, frustración, falta de insumos básicos, información insuficiente y problemas económicos. Conclusión: El afrontamiento del COVID-19 generó un cambio en la forma de vida de la sociedad, por lo que es necesario preparar los servicios de salud para esta demanda, de manera que exista el apoyo psicológico y social y el apoyo de la población en general, dirigiendo la atención a los factores desencadenantes del sufrimiento psíquico, apoyar a las personas en este período y en la pospandémica.
\end{abstract}

Palabras clave: SARS-CoV-2; COVID-19; Coronavirus; Salud mental; Población.

\title{
1. Introdução
}

As pandemias acompanham a humanidade há séculos e são consideradas doenças infecciosas que atingem uma grande região geográfica, espalhando-se para mais de um continente. Essas situações já estiveram presentes em outros momentos da humanidade, como na "Peste Negra", entre 1347-1353, que dizimou 1/3 da população europeia, e na "Gripe Espanhola", que ocorreu entre 1918-1920 (Rezende, 2009).

No início de dezembro de 2019, começaram a ocorrer diversos casos de pneumonia no hospital de Wuhan, na China, chamando a atenção das autoridades locais. Durante esse mês, os números tiveram um aumento importante, levando a notificação para a Organização Mundial de Saúde (OMS) de um cluster de casos de pneumonia por causa desconhecida. Em 30 de janeiro, a OMS declarou surto da doença causada pelo novo coronavírus que constituiu uma Emergência de Saúde Pública de Importância Internacional (ESPII) - o mais alto nível de alerta da Organização Pan-Americana da Saúde (OPAS). COVID19 foi o nome oficial dado pela OMS à doença causada pelo novo coronavírus, COVID significa Corona Virus Disease (Doença do Coronavírus), e “19” se refere a 2019, ano em que os primeiros casos foram divulgados (OMS, 2020; Lana et al., 2020). À medida que houve a progressão da COVID- 19, muitos países adotaram medidas de isolamento social rigoroso, uma quarentena modificada, para reduzir a transmissão do vírus, evitando o contágio da população e consequentemente diminuindo sua propagação e o número dos doentes (Xiao, 2020).

O isolamento social é classificado como um ato voluntário ou involuntário de se manterem os indivíduos isolados do convívio com outros indivíduos ou com a sociedade. $\mathrm{O}$ isolamento social voluntário é aquele em que a pessoa, por conta própria, afasta-se do grupo de convivência e da sociedade por problemas pessoais, tais como depressão ou sentimento de não identificação com ele. O isolamento social involuntário pode ocorrer por outras questões, como a guerra (quando os civis são obrigados a isolar-se em casa ou em abrigos por segurança) e por questões sanitárias (quando, em exemplo do ocorrido devido à pandemia da COVID-19, os indivíduos necessitam isolar-se para que evitar a propagação da doença) (Hortulanus, 2006).

A saúde mental é uma parte integrante e essencial da saúde e não consiste apenas na ausência de doença ou de enfermidade, sendo definida como um estado de bem-estar no qual um indivíduo realiza suas próprias escolhas, lidando com as questões do cotidiano, atuando produtivamente através do trabalho e contribuindo com a sua comunidade. A saúde mental e bem-estar são fundamentais tanto na forma coletiva como individual, e para tal ser desenvolvida e preservada necessita de medidas de promoção e proteção que melhoram o bem-estar psicológico dos indivíduos e populações (OMS, 2013).

O isolamento social ocasionado pela pandemia da COVID- 19 afeta não apenas a saúde física das pessoas, mas também a saúde psicológica e o bem-estar da população não infectada. As ampliações das medidas de isolamento social levaram a população mundial a níveis aumentados de ansiedade, depressão e estresse, sendo necessárias adaptações nos planos 
de curto, médio e longo prazos, que tiveram de ser adiados ou interrompidos (Xiao, 2020). Ao analisar o impacto psicológico do isolamento social em epidemias prévias, verifica-se que os impactos são negativos. Na pandemia de COVID-19, identificase que os principais fatores de estresse estão relacionados à duração do distanciamento social, o medo da contaminação, os sentimentos de frustração e de aborrecimento, as informações inadequadas sobre a doença e seus cuidados, os impactos socioeconômicos e o estigma da doença. Observa-se a ocorrência, nas pessoas em distanciamento social, de sintomas psicológicos, distúrbios emocionais, depressão, estresse, humor depressivo, irritabilidade, insônia e sintomas de estresse póstraumático (Brooks, et al., 2020).

Estudo desenvolvido por Ahmed et al., (2020) na China, primeiro país que adotou a quarentena e o isolamento social como medidas protetivas à disseminação do novo coronavírus, descreveu as consequências psicológicas do isolamento social, em que foi percebido maior índice de ansiedade, depressão, uso nocivo de álcool e menor bem-estar mental que os índices populacionais usuais. Os pacientes com confirmação ou suspeita da COVID-19 apresentaram temor das consequências da infecção, e, de forma geral, toda a população que está realizando o isolamento social refere sentir tédio, solidão e raiva; evidenciando também um acentuado número de situações de estresse ocasionadas pela instabilidade financeira, desencadeada pelo desemprego (Holmes, et al., 2020).

$\mathrm{Na}$ China, estudos também têm reportado prevalências elevadas de depressão e ansiedade no conjunto da população estudada, especialmente em alguns segmentos específicos. Pessoas que precisam respeitar o distanciamento social apresentaram maiores prevalências de depressão e de ansiedade, comparadas aos não afetados pela medida. As pesquisas apontam que indivíduos com transtornos mentais tendem a apresentar níveis mais elevados de estresse e sofrimento psicológico durante a quarentena provocada pela COVID-19, comparados a pessoas sem esses transtornos, em decorrência tanto da maior vulnerabilidade psíquica como de outros fatores; por exemplo, a dificuldade de acesso a tratamento durante a pandemia (Xiao, 2020; (Brooks, et al., 2020).

A letalidade e os prejuízos econômicos no contexto da pandemia geram um alto risco psicossocial. Nesse sentido, a observação da presença de tristeza e ansiedade durante a pandemia, entre pessoas com ou sem transtornos mentais, pode ajudar na definição e/ou orientação de políticas específicas para grupos de risco (Shigemura et al., 2020; Ozamiz-Etxebarria, 2020). Cabe ressaltar que os efeitos para a saúde mental, em geral, são mais marcados nas populações que vivem em condições precárias, possuem recursos escassos e têm acesso limitado aos serviços sociais e de saúde (OMS, 2020).

Assim, considerando-se os efeitos psicológicos da privação de liberdade dos indivíduos que se encontram em isolamento social e considerando que o sofrimento psicológico tende a ser agravado em processos epidêmicos, este estudo teve por objetivo investigar o impacto da pandemia causada pela COVID-19 sobre a saúde mental da população em geral.

\section{Metodologia}

Trata-se de uma revisão integrativa da literatura, que seguiu metodologicamente as seguintes etapas na sua elaboração: (1) delimitação do tema e construção da pergunta norteadora da pesquisa; (2) levantamento das publicações nas bases de dados selecionadas; (3) coleta de dados; (4) classificação e análise das informações achadas em cada material; (5) discussão dos resultados; (6) apresentação dos resultados encontrados e inclusão, análise crítica dos achados e síntese da revisão da literatura (Mendes et al., 2008).

Este estudo tem como questão norteadora: qual o impacto do isolamento social sobre a saúde mental da população geral em tempos de pandemia causada pela COVID-19? Como forma de delimitar a questão norteadora e para direcionar as buscas nas bases de dados, este estudo utilizou-se a estratégia PICO, classificando o objeto de pesquisa da seguinte forma: $\mathrm{P}$ (população) - utilizou-se o descritor "population"; I (intervenção) - utilizaram-se os descritores "coronavirus", "SARS-CoV-2", 
"COVID-19"; O (desfecho) - foi utilizado o descritor "mental health". (Santos et al., 2007). Na sequência, estes descritores foram associados utilizados os operadores booleanos AND e OR com a seguinte estratégia: "population" AND "coronavirus" OR "SARS-CoV-2" OR "COVID-19" AND "mental health". As bases de dados consultadas foram a Medical Literature Analysis and Retrieval System Online (MEDLINE via PubMed), Scientific Electronic Library Online (SciELO), Web of Science (WoS), Service Scopus (SCOPUS), Biblioteca Virtual em Saúde (BVS) e PsycoInfo.

Os estudos foram localizados por uma busca avançada, o levantamento de dados foi iniciado em maio até dezembro de 2020, e foram utilizados filtros de três idiomas (português, inglês, espanhol) e com data de publicação no ano de 2020. Foram considerados elegíveis artigos originais e publicados em periódicos nacionais e internacionais, com data de publicação originária do ano de 2020, disponíveis em língua portuguesa, inglesa e espanhola que contemplassem a questão norteadora. Foram excluídos manuscritos que não responderam ao objetivo do estudo, assim como os resultantes de artigos de revisão, editoriais, comentários, livros, relatos de experiência, preprint e artigos em duplicidade.

\section{Resultados}

Para sistematizar o processo de seleção dos artigos optou-se pela metodologia Preferred Reporting Items for Systematic Reviews and Meta-Analyses (PRISMA) (Moher et al, 2009). As etapas desse processo estão descritas na forma de um fluxograma (Figura 1). Foram identificados 1538 artigos nas bases de dados mencionadas anteriormente, e, verificados os manuscritos pelo título, pelo resumo e por duplicidade, foram excluídos 606 artigos, restando 932 trabalhos. Posteriormente, foi realizada a aplicabilidade dos critérios de inclusão e de exclusão, resultando na exclusão de 303 artigos. Feitos o refinamento e a aplicação da questão norteadora, houve exclusão de 257, restando para a leitura na íntegra 46 artigos. Após a leitura na íntegra esses artigos e a avaliação final dos estudos, foram selecionados 16 artigos que compõem esta revisão integrativa. Após a análise crítica da literatura selecionada, foi construído o Quadro 1 para condensar as evidências encontradas. As dimensões de análise foram as seguintes: autores, objetivos, dados de delineamento metodológico e resultados. 
Research, Society and Development, v. 10, n. 4, e52010414411, 2021

(CC BY 4.0) | ISSN 2525-3409 | DOI: http://dx.doi.org/10.33448/rsd-v10i4.14411

Figura 1 - Fluxograma de Prisma.
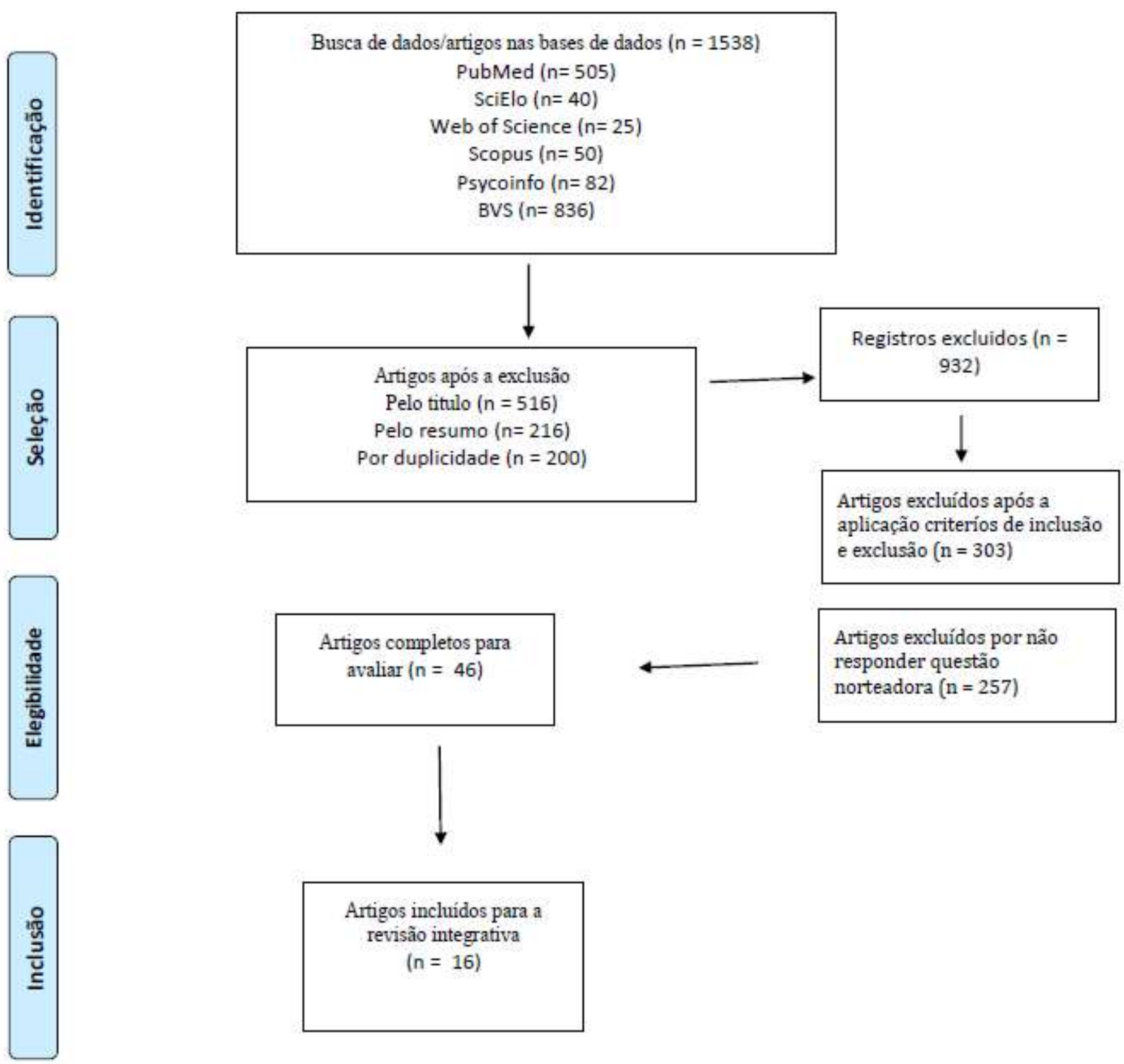

Artigos excluídos por não responder questão norteadora $(\mathrm{n}=257)$

Fonte: Autores (2021). 
Quadro 1 - Autores, objetivos, delineamento metodológico e principais resultados.

\begin{tabular}{|c|c|c|c|}
\hline Autores & Objetivos & Método do estudo & Resultados \\
\hline $\begin{array}{l}\text { Andrés L. B, et } \\
\text { al. }\end{array}$ & $\begin{array}{l}\text { Analisar ansiedade, } \\
\text { tristeza e solidão em } \\
\text { jovens, meia-idade e } \\
\text { idosos confinados } \\
\text { devido à pandemia de } \\
\text { COVID-19. }\end{array}$ & $\begin{array}{l}\text { Pesquisa quantitativa, desenvolvida } \\
\text { por questionário elaborado pelos } \\
\text { pesquisadores e divulgado por meio } \\
\text { do Google Forms, aplicado em } \\
\text { março de 2020, em adultos } \\
\text { espanhóis com faixa etária de } 18 \text { a } \\
88 \text { anos. }\end{array}$ & $\begin{array}{l}\text { Quanto mais jovem o adulto, maior seu nível de } \\
\text { ansiedade. Os de meia-idade relatam menos } \\
\text { solidão, seguidos pelos idosos e jovens. As } \\
\text { comorbidades depressão e ansiedade aparecem } \\
\text { com mais frequência em jovens e menos em } \\
\text { pessoas mais velhas; há mais pessoas deprimidas } \\
\text { na meia-idade, e menos idosos com ansiedade e } \\
\text { sintomas depressivos. }\end{array}$ \\
\hline $\begin{array}{l}\text { Allen C. S, et } \\
\text { al. }\end{array}$ & $\begin{array}{l}\text { Examinar o impacto } \\
\text { da pandemia na saúde } \\
\text { mental e morbidade } \\
\text { psicossocial } \\
\text { Arkansas. }\end{array}$ & $\begin{array}{l}\text { Estudo transversal nos Estados } \\
\text { Unidos, que trata de sintomas } \\
\text { depressivos (PHQ-9), ansiedade } \\
\text { generalizada (GAD-7), sintomas } \\
\text { relacionados ao trauma (PCL-5) e } \\
\text { uso de álcool (AUDIT-C). }\end{array}$ & $\begin{array}{l}\text { O estudo se concentrou em um período de } \\
\text { reabertura gradual, mas com taxas de infecção } \\
\text { crescentes nos Estados Unidos, no qual } 21 \% \text { dos } \\
\text { participantes relataram níveis clinicamente } \\
\text { elevados de sintomas depressivos no PHQ-9, } \\
16,58 \% \text { relataram sintomas de ansiedade } \\
\text { generalizada elevada no GAD-7 e 5,38\% } \\
\text { apresentaram níveis elevados de sintomas de } \\
\text { trauma no PCL-5. O uso indevido de álcool } \\
\text { potencial foi relatado por } 35,03 \% \text { (35,59\% das } \\
\text { mulheres e } 33,08 \% \text { dos homens) no AUDIT-C. }\end{array}$ \\
\hline Alomo M, et al. & $\begin{array}{l}\text { Impacto psicológico } \\
\text { da pandemia e do } \\
\text { confinamento } \\
\text { preventivo na } \\
\text { população argentina. }\end{array}$ & $\begin{array}{l}\text { Estudo observacional quantitativo, } \\
\text { na população adulta da } \\
\text { Argentina, no mês de abril de } \\
2020 \text { Utilizando questionário } \\
\text { sociodemográfico e a escala de } \\
\text { medo da contaminação pela } \\
\text { COVID- 19, }\end{array}$ & $\begin{array}{l}\text { O medo da COVID- } 19 \text { foi maior nas pessoas de } \\
55 \text { a } 59 \text { anos, com queda abrupta acima de } 70 \text {; o } \\
\text { humor deprimido e o aumento do consumo de } \\
\text { álcool foram mais frequentes nos indivíduos mais } \\
\text { jovens, e o humor deprimido também foi } \\
\text { frequente entre os indivíduos que perderam o } \\
\text { emprego durante a quarentena. Há elevado grau } \\
\text { de concordância com a medida de quarentena, } \\
\text { mesmo em algumas pessoas que sofreram um } \\
\text { decréscimo em sua renda superior a } 80 \% \text { neste } \\
\text { período. }\end{array}$ \\
\hline $\begin{array}{l}\text { Atchimson } \mathrm{C}, \text { et } \\
\text { al. }\end{array}$ & $\begin{array}{l}\text { Percepções de risco e } \\
\text { respostas } \\
\text { comportamentais das } \\
\text { estratégias de controle } \\
\text { da COVID-19. }\end{array}$ & $\begin{array}{l}\text { Estudo transversal quantitativo, } \\
\text { realizado com } 2.108 \text { adultos do } \\
\text { Reino Unido com } 18 \text { anos ou mais. } \\
\text { Os dados foram coletados entre } 17 \mathrm{e} \\
18 \text { de março de } 2020 \text {, através de } \\
\text { questionário online. }\end{array}$ & $\begin{array}{l}\text { Da amostra total da pesquisa } 94,2 \% \text { dos } \\
\text { entrevistados relataram tomar pelo menos uma } \\
\text { medida preventiva; } 85,8 \% \text { lavaram as mãos com } \\
\text { sabonete com mais frequência; } 56,5 \% \text { evitaram } \\
\text { áreas lotadas e } 54,5 \% \text { evitaram eventos sociais. A } \\
\text { adoção de medidas de distanciamento foi maior } \\
\text { naqueles com mais de } 70 \text { anos em comparação } \\
\text { com os adultos mais jovens (18 a } 34 \text { anos); } \\
\text { pessoas com renda familiar mais baixa } \\
\text { apresentaram seis vezes menos probabilidade de } \\
\text { conseguir trabalhar em casa e três vezes menos } \\
\text { de conseguirem se isolar, o que também foi } \\
\text { menor em grupos étnicos negros. Em ambos } \\
\text { grupos houve um desejo para o isolamento social } \\
\text { e compreensão do motivo deste isolamento. }\end{array}$ \\
\hline $\begin{array}{l}\text { Bezerra, A. C, } \\
\text { et al. }\end{array}$ & $\begin{array}{l}\text { Descrever, a } \\
\text { percepção das pessoas } \\
\text { sobre como sua vida é } \\
\text { afetada pelo } \\
\text { isolamento social }\end{array}$ & $\begin{array}{l}\text { Estudo qualitativo, (Google Forms), } \\
\text { via redes sociais, com } 17 \text { questões } \\
\text { (pesquisa de opinião), realizada em } \\
\text { abril de } 2020 \text {, com jovens adultos } \\
\text { brasileiros. Obteve-se uma amostra } \\
\text { com } 16.440 \text { respondentes. }\end{array}$ & $\begin{array}{l}\text { O convívio social foi o aspecto mais afetado entre } \\
\text { pessoas com maior escolaridade e renda }(45,8 \%) \text {. } \\
\text { Para pessoas de baixa renda e escolaridade, } \\
\text { problemas financeiros provocam maior impacto } \\
(35 \%) \text {. Os que praticam atividade física } \\
\text { revelaram menores níveis de estresse }(13 \%) \text {, bem } \\
\text { como melhor sono }(50,3 \%) \text {. Quem reside em } \\
\text { piores condições informou disposição a } \\
\text { permanecer menos tempo isolado (73,9\%). }\end{array}$ \\
\hline
\end{tabular}




\begin{tabular}{|c|c|c|c|}
\hline & & & $\begin{array}{l}\text { Dentre as pessoas não isoladas ( } 10,7 \% \text { do total), } \\
75,8 \% \text { acredita que o isolamento reduzirá o } \\
\text { número de vítimas. A percepção quanto ao } \\
\text { isolamento social varia conforme a renda, } \\
\text { escolaridade, idade e sexo, porém a maior parte } \\
\text { concorda com a medida e estão dispostas a } \\
\text { contribuir com o enfrentamento à COVID- } 19 \text {. }\end{array}$ \\
\hline $\begin{array}{l}\text { Barros M B.A, } \\
\text { et al. }\end{array}$ & $\begin{array}{lr}\text { Analisar a frequência } \\
\text { de } & \text { tristeza, } \\
\text { nervosismo } & \text { e } \\
\text { alterações do sono } \\
\text { durante a pandemia } \\
\text { de COVID-19 no } \\
\text { Brasil. }\end{array}$ & $\begin{array}{l}\text { Estudo transversal quantitativo, com } \\
\text { questionário, utilizando aplicativo } \\
\text { RedCap (Research Eletronic Data } \\
\text { Capture), no período de abril a } \\
\text { maio de } 2020 \text {, na população adulta } \\
\text { brasileira. }\end{array}$ & $\begin{array}{l}\text { Dos } 45.161 \text { brasileiros respondentes, verificou-se } \\
\text { que } 40,4 \% \text { se sentiram tristes ou deprimidos, } \\
52,6 \% \text { ansiosos ou nervosos; } 43,5 \% \text { relataram } \\
\text { início de problemas de sono, } 48,0 \% \text { problemas de } \\
\text { sono preexistentes agravados. Tristeza, } \\
\text { nervosismo frequentes e alterações do sono } \\
\text { estavam mais presentes entre adultos jovens, } \\
\text { mulheres e pessoas com antecedentes de } \\
\text { depressão. }\end{array}$ \\
\hline $\begin{array}{l}\text { Chakraborty, } \\
\text { K., \& } \\
\text { Chatterjee, M. }\end{array}$ & $\begin{array}{l}\text { Avaliar o impacto } \\
\text { psicológico } r \text { da } \\
\text { pandemia COVID-19 } \\
\text { na população em geral } \\
\text { em West Bengal. }\end{array}$ & $\begin{array}{l}\text { Pesquisa quantitativa, com } \\
\text { questionário auto-elaborado que } \\
\text { incluiu perguntas sobre perfil } \\
\text { sociodemográfico, conhecimento e } \\
\text { atitudes em relação à pandemia e o } \\
\text { aspecto psicológico da pandemia } \\
\text { COVID-19. Realizado em março de } \\
\text { 2020, com } \mathbf{5 0 7} \text { jovens adultos na } \\
\text { Índia. }\end{array}$ & $\begin{array}{l}\text { Do total de entrevistados, } 71,8 \% \text { estavam } \\
\text { preocupados com a pandemia e } 24,7 \% \text { estavam } \\
\text { deprimidos nas duas semanas anteriores ao } \\
\text { estudo, } 52,1 \% \text { relataram preocupação com a ideia } \\
\text { de contaminação e } 21,1 \% \text { pensavam } \\
\text { repetidamente em fazer o teste de presença de } \\
\text { COVID- } 19 \text {, apesar de não apresentarem } \\
\text { sintomas; } 69,6 \% \text { estavam preocupados com a } \\
\text { instabilidade e perdas financeiras que estavam } \\
\text { sofrendo durante o período; } 25,6 \% \text { descobriram } \\
\text { que a pandemia havia ameaçado sua existência e } \\
30,8 \% \text { tiveram dificuldades em se adaptar à nova } \\
\text { rotina de restrições. }\end{array}$ \\
\hline $\begin{array}{l}\text { Delmastro M; } \\
\text { Zamariola, G. }\end{array}$ & $\begin{array}{lr}\text { Avaliar o } & \text { impacto } \\
\text { psicológico } & \text { da } \\
\text { COVID-19 } & \text { na } \\
\text { população italiana. }\end{array}$ & $\begin{array}{l}\text { Pesquisa quantitativa de junho de } \\
2020 \text { com italianos jovens adultos } \\
\text { (16-24) e adultos (25+), por meio } \\
\text { do instrumento Short Mood and } \\
\text { Feelings Questionnaire (SMFQ). }\end{array}$ & $\begin{array}{l}\text { Dos } 6.700 \text { italianos entrevistados, os sintomas } \\
\text { depressivos aparecem mais em mulheres, adultos } \\
\text { jovens, pessoas com incerteza profissional e } \\
\text { status socioeconômico inferior. Quem mora } \\
\text { sozinho sofre menos com o isolamento. }\end{array}$ \\
\hline $\begin{array}{l}\text { Duarte M. Q, et } \\
\text { al. }\end{array}$ & $\begin{array}{l}\text { Transtornos mentais } \\
\text { em residentes do Rio } \\
\text { Grande do Sul, } \\
\text { durante o período } \\
\text { inicial da pandemia da } \\
\text { COVID-19. }\end{array}$ & 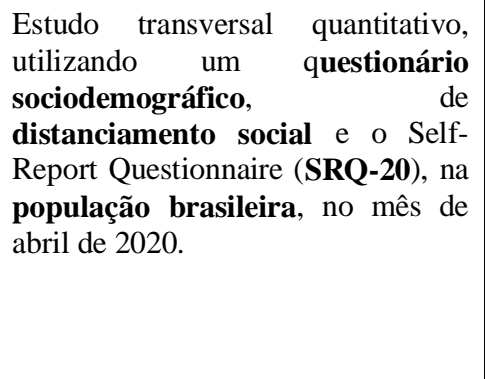 & $\begin{array}{l}\text { Participaram } 799 \text { pessoas, com idades entre } 18 \text { e } \\
75 \text { anos. Os resultados indicaram que ter renda } \\
\text { diminuída no período, fazer parte do grupo de } \\
\text { risco e estar mais exposto a informações sobre } \\
\text { mortos e infectados, são fatores que podem } \\
\text { provocar maior prejuízo na saúde mental neste } \\
\text { período de pandemia. Investigar determinantes } \\
\text { sociais sobre a vulnerabilidade ao adoecimento } \\
\text { mental é importante para o planejamento de } \\
\text { ações e políticas públicas. }\end{array}$ \\
\hline $\begin{array}{l}\text { Enumo S. R. F, } \\
\text { et al. }\end{array}$ & $\begin{array}{l}\text { Elaborar cartilha de } \\
\text { orientação aos } \\
\text { cuidados em saúde, na } \\
\text { pandemia de COVID- } \\
19 .\end{array}$ & $\begin{array}{l}\text { Pesquisa qualitativa, online, } \\
\text { desenvolvida pelos pesquisadores. } \\
\text { Período de análise abril de } 2020, \\
\text { população entrevistada adultos } \\
\text { brasileiros, entre } 28 \text { a } \mathbf{6 5} \text { anos de } \\
\text { idade. }\end{array}$ & $\begin{array}{l}\text { Após a análise dos oito entrevistados, relacionado } \\
\text { à cartilha de cuidado de saúde em tempos de } \\
\text { pandemia, obteve-se o seguinte resultado: a } \\
\text { cartilha fundamentada em conceitos psicológicos } \\
\text { bem estabelecidos poderá auxiliar no } \\
\text { enfrentamento do estresse, orientando sobre } \\
\text { como lidar com os problemas emocionais e } \\
\text { comportamentais relacionados à pandemia de } \\
\text { COVID-19. }\end{array}$ \\
\hline $\begin{array}{l}\text { Johnson M. C, } \\
\text { et al. }\end{array}$ & $\begin{array}{lr}\text { Conhecer } & \text { os } \\
\text { sentimentos } & \text { das } \\
\text { populações } & \text { frente } \\
\text { COVID-19. } & \end{array}$ & $\begin{array}{l}\text { Pesquisa qualitativa, desenvolvida } \\
\text { via questionário elaborado pelos } \\
\text { pesquisadores no Google Form, } \\
\text { divulgado via redes sociais. }\end{array}$ & $\begin{array}{l}\text { A população pesquisada apresentou incerteza, } \\
\text { medo e angústia, mas também apresenta } \\
\text { sentimento de responsabilidade e cuidado diante } \\
\text { (COVID-19). O impacto na saúde mental é }\end{array}$ \\
\hline
\end{tabular}




\begin{tabular}{|c|c|c|c|}
\hline & & $\begin{array}{l}\text { Amostra total de } 1.410 \\
\text { entrevistados, na Argentina, de } \\
\text { março a abril de } \mathbf{2 0 2 0} \text {. }\end{array}$ & $\begin{array}{l}\text { desigual em função de gênero, escolaridade } \\
\text { alcançada e o conforto percebido no domicílio. }\end{array}$ \\
\hline Lu P, et al. & $\begin{array}{lr}\text { Avaliar a saúde mental } \\
\text { das pessoas e } & \text { as } \\
\text { mudanças depois } & \text { que } \\
\text { Wuhan } & \text { realizou } \\
\text { medidas } & \text { de } \\
\text { relaxamento } & \text { das } \\
\text { medidas } & \text { de } \\
\text { distanciamento. } & \end{array}$ & $\begin{array}{l}\text { Estudo transversal quantitativo com } \\
\text { o Questionário online de saúde do } \\
\text { paciente-9 (PHQ-9), Transtorno de } \\
\text { Ansiedade Generalizada de } 7 \text { itens } \\
\text { (GAD-7) e o PTSD Checklist- } \\
\text { Civilian Version (PCL-C), na } \\
\text { população chinesa, no mês de } \\
\text { junho de 2020, com uma amostra de } \\
1417 \text { participantes. }\end{array}$ & $\begin{array}{l}\text { Dos } 387 \text { entrevistados }(27,0 \%) \text { eram profissionais } \\
\text { de saúde de linha de frente e } 1.035(73,0 \%) \\
\text { população em geral. } \\
\text { Dos } 73 \% \text { da população geral, } 46 \% \text { apresentaram } \\
\text { sintomas de depressão, ansiedade e TEPT } \\
\text { variando de moderado a grave. A diminuição da } \\
\text { renda e a exposição frequente às redes sociais são } \\
\text { fatores de risco para o desenvolvimento de } \\
\text { alterações mentais para o público geral. }\end{array}$ \\
\hline Roy D, et al. & $\begin{array}{l}\text { Avaliar ansiedade e a } \\
\text { percepção da } \\
\text { necessidade de saúde } \\
\text { mental durante a } \\
\text { pandemia de COVID- } \\
19 .\end{array}$ & $\begin{array}{l}\text { Pesquisa quantitativa, online por } \\
\text { questionário semiestruturado na } \\
\text { população indiana adulta, com } \\
662 \text { respondentes. }\end{array}$ & $\begin{array}{l}\text { Das pessoas entrevistadas } 80 \% \text { estavam } \\
\text { preocupadas com a COVID-19 e } 72 \% \text { relataram a } \\
\text { necessidade de usar luvas e desinfetantes. Neste } \\
\text { estudo, } 12,5 \% \text { dos participantes apresentaram } \\
\text { dificuldades para dormir, } 37,8 \% \text {, paranóia sobre a } \\
\text { aquisição da infecção e mídia social relacionada à } \\
\text { angústia foram relatados em } 36,4 \% \text {. A percepção } \\
\text { da necessidade de saúde mental foi observada em } \\
80 \% \text { dos participantes. }\end{array}$ \\
\hline Wang C, et al. & $\begin{array}{l}\text { Compreender os } \\
\text { impactos } \\
\text { psicológicos, } \\
\text { ansiedade, depressão } \\
\text { e estresse durante o } \\
\text { estágio inicial do surto } \\
\text { COVID-19, na } \\
\text { população chinesa. }\end{array}$ & $\begin{array}{l}\text { Pesquisa quantitativa, realizada na } \\
\text { China, de janeiro a fevereiro de } \\
2020 . \\
\text { sociodemográfico; o Impact of } \\
\text { Event Scale-Revised (IES-R) e a } \\
\text { Escala de Depressão, Ansiedade e } \\
\text { Estresse (DASS-21). }\end{array}$ & $\begin{array}{l}\text { Ao total foram entrevistados } 1.210 \text { de } 194 \\
\text { cidades na China. } 53,8 \% \text { dos entrevistados } \\
\text { classificaram o impacto psicológico como } \\
\text { moderado ou grave; } 16,5 \% \text { relataram sintomas } \\
\text { depressivos; } 28,8 \% \text { relataram sintomas de } \\
\text { ansiedades; e } 8,1 \% \text { relataram níveis de estresse. } \\
\text { A maioria dos entrevistados passou de } 20 \text { a } 24 \\
\text { horas em casa }(84,7 \%) \text {; preocupavam-se com a } \\
\text { possibilidade de seus familiares contaminarem } \\
\text { pela COVID-19 (75,2\%); e ficaram satisfeitos } \\
\text { com a quantidade de informações de saúde } \\
\text { disponíveis }(75,1 \%) \text {. }\end{array}$ \\
\hline Wang $\mathrm{C}$, et al. & $\begin{array}{l}\text { Impacto psicológico, } \\
\text { ansiedade, depressão } \\
\text { e estresse durante o } \\
\text { estágio inicial do } \\
\text { surto COVID-19. }\end{array}$ & $\begin{array}{l}\text { Pesquisa quantitativa, questionário } \\
\text { online, por amostragem em bola de } \\
\text { neve, realizado com jovens adultos } \\
\text { na China em fevereiro a março de } \\
\text { 2020, usando o Impact of Event } \\
\text { Scale-Revised (IES-R) e a Escala } \\
\text { de Depressão, Ansiedade e Estresse } \\
\text { (DASS-21). }\end{array}$ & $\begin{array}{l}\text { Mais da metade dos entrevistados classificou o } \\
\text { impacto psicológico como moderado a grave e } \\
\text { cerca de um terço relatou ansiedade moderada a } \\
\text { grave. Os resultados identificaram fatores } \\
\text { associados a um menor nível de impacto } \\
\text { psicológico e melhor estado de saúde mental, } \\
\text { sendo este impacto percebido devido ao declínio } \\
\text { no número de casos novos e suspeitos, e a } \\
\text { tendência de queda dos infectados pela COVID- } \\
\text { 19. Simultaneamente, o número de pacientes } \\
\text { recuperados apresentou um aumento substancial } \\
\text { durante este período. }\end{array}$ \\
\hline Zhang J, et al. & $\begin{array}{l}\text { Identificar } \\
\text { sofrimento } \\
\text { psicológico da } \\
\text { população durante o } \\
\text { período de pandemia } \\
\text { pela COVID- } 19 .\end{array}$ & $\begin{array}{l}\text { Pesquisa quantitativa realizada por } \\
\text { plataformas virtuais, aplicando os } \\
\text { questionários PHQ- } 9 \text { E GAD- 7, } \\
\text { em fevereiro de 2020, na } \\
\text { população adulta chinesa. }\end{array}$ & $\begin{array}{l}\text { Dos } 205 \text { participantes identificou- se que } \mathbf{2 9 , 2 \%} \\
\text { apresentaram maior prevalência para } \\
\text { sintomas depressivos, sendo os pacientes que } \\
\text { foram infectados pela COVID-19 } 19,3 \% \text { e o } \\
\text { público geral, } 14,3 \% \text {, às crises de ansiedade } \\
\text { foram identificados em pacientes que sofreram } \\
\text { infecção por COVID-19 em } 21,1 \% \text { e no público } \\
\text { geral em } 22,4 \% \text {. }\end{array}$ \\
\hline
\end{tabular}

Fonte: Autores (2021). 


\section{Discussão}

Com a pandemia ocasionada pela COVID-19, tem-se percebido um impacto psicológico imediato, sendo observado um crescimento de sintomas de ansiedade e de depressão (Wang et al., 2020). Além disso, tanto pelo fato da ameaça em si quanto pela adoção de medidas de enfrentamento, ocorreu uma alteração significativa dos modos de viver dos indivíduos, assim como houve rupturas importantes nas relações sociais (Andrés et al., 2020).

Um dos principais gatilhos para o surgimento do estresse durante a pandemia é o sentimento de perda do direito de ir e vir, que ocasiona um estado de negação da gravidade da doença. Isso leva automaticamente à desconsideração da relevância do isolamento social por meio de um autoboicote inconsciente de atitudes e de comportamentos individuais, como a ausência de higienização das mãos, o uso de máscara, as aglomerações e as saídas de casa (Enumo, et al., 2020). O sentimento de incerteza relacionado ao estresse e aos limites impostos pelas medidas preventivas de isolamento social, havendo a hipótese de alterações, de modo drástico, nos planos, além da separação brusca do ambiente social ou familiar do indivíduo, tornam-se catalisadores constantes para o surgimento de sintomas de ansiedade e até mesmo de depressão (Lu et al., 2020).

Pesquisas realizadas na China, no Brasil, na Itália, na Índia, na Argentina, na Espanha e nos Estados Unidos (Lu et al., 2020; Duarte et al., 2020; Delmastro \& Zamariola, 2020; Roy et al., 2020; Andrés et al., 2020; Allen et al., 2020) mostram que a pandemia COVID-19 afetou a saúde mental, revelando a presença de sintomas de ansiedade, depressão e estresse autorreferido associados a distúrbios do sono. Variáveis como ser do sexo feminino, ser estudante, apresentar sintomas sugestivos de COVID-19 e percepção de saúde ruim foram associadas a maiores taxas de ansiedade, insônia e depressão. Sendo assim, esses segmentos demográficos sinalizam maior vulnerabilidade, portanto, demandam a aplicação e o aprimoramento de estratégias de preservação e de atenção à saúde mental durante a pandemia. Outras características que contribuíram para o estresse e para a morbidade mental foram a imprevisibilidade, a incerteza, a gravidade da doença, a desinformação e o isolamento social.

Para a apresentação e a discussão dos resultados, as pesquisas incluídas neste estudo utilizaram diferentes instrumentos para coleta de dados, entre eles o Impact of Event Scale -Revised (IES -R), Depression, Anxiety and Stress Scale (DASS), Generalized Anxiety Disorder - 7 Scale (GAD -7), Short Mood and Feelings Questionnaire (SMFQ), Patient Health Questionnaire-9. (PHQ-9) e Post-Traumatic Stress Disorder Checklist - Civilian Version (PCL-C). Esses instrumentos foram importantes para a avaliação dos transtornos que estão acontecendo na saúde mental das populações investigadas em tempo de pandemia (Delmastro \& Zamariola, 2020; Lu et al., 2020; Wang et al., 2020; Allen et al., 2020; Zhang et al., 2020; Wang et al., 2020; Duarte et al., 2020).

Os resultados da pesquisa de Barros et al. (2020) mostram que, durante o período da pandemia estudado, dos 45.161 respondentes, 40,4\% dos adultos brasileiros sentiram-se tristes ou deprimidos, e 52,6\% reportaram estarem ansiosos ou nervosos. Entre os que não tinham problemas de sono, mais de $40 \%$ passaram a ter, e quase $50 \%$ dos que já tinham tiveram o problema agravado. Os sentimentos de tristeza e de ansiedade e os problemas do sono revelaram prevalências mais elevadas em adultos jovens, mulheres e pessoas com diagnóstico prévio de depressão.

Em outro estudo (Lu et al., 2020) realizado na China, os percentuais têm a seguinte representatividade: $21,2 \%$ dos entrevistados mencionaram estar depressivos, 16,7\% referiram estar ansiosos, e 17,2\% mencionaram transtorno de estresse pós-traumático, variando de moderado a grave. Nesse mesmo estudo, a diminuição da renda e a exposição frequente às redes sociais foram identificadas como fatores de risco para o público em geral. Os resultados são semelhantes em Wuhan, na China, com um percentual de $46 \%$ de sintomas depressivos na população (Wang et al., 2020).

$\mathrm{Na}$ Índia, os resultados relacionados à insônia e à angústia foram relatados em 12,5\% e 36,4\% dos participantes, respectivamente, e a percepção da necessidade de saúde mental foi observada em $80 \%$ dos participantes (Roy et al., 2020). No 
entanto, a pesquisa brasileira de Barros et al. (2020) revelou que 52,6\%, dos entrevistados mencionaram que frequentemente encontram-se ansiosos ou nervosos, 43,5\% mencionaram problemas de insônia, e 48,0\% relataram que seus problemas de sono preexistentes foram agravados, no período de isolamento social.

Seguindo esse panorama de dados, o estudo de Chakraborty \& Chatterjee (2020), desenvolvido na Índia com 507 entrevistados, identificou que $(71,8 \%)$ e $(24,7 \%)$ sentiram-se mais preocupados e deprimidos, respectivamente, e metade $(52,1 \%)$ estava preocupada com a ideia de contaminação pela COVID-19. Além das consequências da contaminação e as alterações na saúde mental, a maioria $(69,6 \%)$ dos entrevistados estava preocupada com a instabilidade e as perdas financeiras que estavam sofrendo durante o período de bloqueio. Um quarto $(25,6 \%)$ e um terço $(30,8 \%)$ dos entrevistados descobriram que a pandemia de COVID-19 havia ameaçado sua existência, e eles tiveram dificuldades em se adaptar à nova rotina de restrições.

Pesquisa de Wang et al. (2020) realizada na China, com 1.210 participantes evidenciou que 53,8\% da população sofreu impactos psicológicos de intensidade moderada a grave na pandemia da COVID-19. Os principais efeitos desses impactos ocasionados pela COVID-19 foram observados em pessoas que já apresentavam alguma vulnerabilidade de sintomas depressivos e nos profissionais de saúde. Em paralelo a esta discussão, o estudo realizado na Argentina (Johnson et al. 2020) discute a importância de se conhecerem os riscos para o surgimento de transtornos mentais, além de disponibilizar programas de intervenções psicoterapêuticas adaptáveis e dinâmicas ao longo da pandemia, sendo necessário articular ações de suporte de forma direcionada às especificidades das demandas, disponibilizando serviços on-line para atenção a pacientes necessitados de cuidados quanto às suas condições emocionais e mentais.

$\mathrm{Na}$ Espanha, a pesquisa de Andrés et al. (2020) desenvolvida com adultos na faixa etária compreendida entre 18 a 88 anos evidenciou que pessoas mais velhas apresentaram menos ansiedade e tristeza, sendo referido que $12,52 \%$ se encontravam ansiosos; $8,21 \%$, tristes, e $33,03 \%$, estavam solitários, enquanto no público de meia-idade e jovem estes percentuais foram de $31,35 \%$ para ansiedade, $24,79 \%$ e para tristeza e 40,40\% para solidão, respectivamente, sendo possível observar que há mais pessoas deprimidas na meia-idade, e menos idosos com ansiedade e sintomas depressivos.

Como visto, a pandemia de COVID-19 tem ocasionado diversos agentes estressores, incluindo solidão decorrente do isolamento social, medo de contrair a doença, tensão econômica e incerteza sobre o futuro. Embora esses elementos sejam evidentes na sociedade toda, a população idosa vem enfrentando os impactos de maneira mais resiliente, pois não foi atingida na parte financeira por estar aposentada e a relação da vida social ser menos intensa e agitada. Esses idosos, em comparação com os mais jovens, sofreram menos com a privação das atividades sociais. Entre os adultos mais jovens, a necessidade de contato social pode estar contribuindo para o maior abalo à saúde emocional desse grupo (Andrés et al., 2020; Barros et al., 2020).

Os estudos de Delmastro \& Zamariola (2020), Lu et al. (2020) e Zhang et al. (2020) evidenciam que ocorrerão graves consequências na vida da população devido à COVID-19 nos próximos meses e anos, relacionadas às alterações físicas e mentais. Nesse sentido, deve-se considerar a inserção de cuidados na saúde da população geral, mediante realização de grupos virtuais, acompanhamento individual de forma remota, estímulo a atividades físicas e avaliação da equipe para acompanhamentos presenciais, quando necessários. Esses cuidados poderão diminuir os impactos do momento atual e estabelecer intervenções psicológicas que fortaleçam a capacidade mental dos indivíduos, com ações e serviços individualizados e coletivos.

Destaca-se que as intervenções psicológicas deverão estar alicerçadas em três eixos principais: compreensão do estado da saúde mental em diferentes populações influenciadas pelo surto de COVID- 19; identificação de pessoas com alto risco de suicídio e fortalecimento de intervenções psicológicas adequadas para os necessitados; e o fortalecimento dos vínculos afetivos que foram quebrados durante o período do isolamento (Lu et al., 2020; Zhang et al., 2020). A abordagem da Terapia Cognitiva 
Comportamental (TCC), por intermédio da educação psicológica, tem sido uma estratégia de abordagem que permite ensinar o paciente a lidar com a doença, seja ela física ou mental, sendo uma eficiente intervenção para a população geral. Outras técnicas de estímulo da TCC, como relaxamento e respiração para o controle da ansiedade, colaboram para a manutenção da saúde mental e física (Lu et al., 2020).

Para este enfrentamento também se faz necessária a compreensão de como a população vem lidando com o isolamento social e como isso tem impactado nas suas vidas. (Duarte et al., 2020). Para uma melhor adesão às práticas de isolamento e de enfrentamento das condições geradas por isso, a população precisa estar informada sobre os resultados positivos dessas atitudes com o objetivo de impulsionar a sua adesão. Isso pode ter refletido na implementação bem sucedida das medidas de isolamento social pela população da China, com as quais foi possível erradicar a proliferação do vírus e a diminuição considerável de novos casos de COVID-19 no país (Zhang et al., 2020). Bezzerra et al. (2020) destacam que a percepção das pessoas quanto ao isolamento social varia conforme a renda, a escolaridade, a idade e o sexo, porém a maior parte dos entrevistados acreditam que se trata da medida de controle mais indicada e estão dispostas a esperar o tempo que for necessário para contribuir com o enfrentamento à COVID-19.

No Reino Unido, os principais resultados apresentados pela adoção de medidas de isolamento evidenciaram que 56,5\% dos entrevistados evitaram áreas lotadas, e 54,5\% evitaram eventos sociais. A adoção de medidas de distanciamento social foi maior naqueles indivíduos com mais de 70 anos, em comparação com os adultos mais jovens de 18 a 34 anos; pessoas com renda familiar mais baixa apresentaram seis vezes menos probabilidade de conseguir trabalhar em casa e três vezes menos probabilidade de conseguirem se isolar. A capacidade de isolar-se também foi menor em grupos étnicos negros, porém, em ambos os grupos entrevistados, houve um desejo individual para o isolamento social e a compreensão do motivo deste isolamento (Atchimson et al., 2020). Duarte et al, (2020) indicaram que ter renda diminuída no período, fazer parte do grupo de risco e estar mais exposto a informações sobre mortos e infectados são fatores que podem provocar maior prejuízo na saúde mental neste período de pandemia. Investigar os determinantes sociais que contribuem para a maior vulnerabilidade ao adoecimento mental da população é importante no campo da saúde coletiva para o planejamento de ações e políticas públicas.

A pandemia da COVID-19 colocou tudo em segundo plano, condicionando e mudando a dinâmica das relações da sociedade italiana, segundo país mais afetado pela pandemia, que, além de todo o estresse a que foram submetidos, relacionado ao desafio sem precedentes ao cenário de saúde geral desta população, a saúde mental, também sofreu seus impactos, com um aumento na ansiedade, na depressão e nos sentimentos de angústia, sintomas mais prevalentes em mulheres jovens. Os entrevistados do estudo italiano relataram incerteza na área profissional e no status socioeconômico e reforçam a importância de atuar nos efeitos psicológicos da pandemia, fornecendo suporte aos indivíduos que buscam atendimento em saúde mental (Delmastro \& Zamariola, 2020). A pesquisa realizada na Índia apresentou evidenciou que a maioria dos entrevistados se sentia preocupada com a restrição financeira durante as medidas de bloqueio, quase um quarto experimentou sintomas depressivos, e um terço achou difícil se ajustar ao novo cotidiano; $25,6 \%$ dos entrevistados consideraram que a COVID-19 havia ameaçado sua existência (Chakraborty \& Chatterjee, 2020).

Pessoas diagnosticadas com COVID-19 também estão suscetíveis a algum sofrimento mental, o que pode gerar reações como angústia, medo, tédio, solidão, insônia ou raiva. Acresce-se a isso o aumento nos comportamentos de risco à saúde como o aumento do consumo de álcool, cigarro e outras drogas nesse período (Andrés et al., 2020). Portanto, é preciso separar os efeitos psicológicos produzidos na situação de pandemia de COVID-19, isto é, compreendê-los e contextualizá-los, além de prover a redução dos danos de vulnerabilidades e atuar nas demandas particulares de cada população (Wang et al.,2020; Bezerra et al., 2020). 
Em paralelo a essa discussão, estudos realizados por Johnson et al. (2020) e Alomo et al. (2020) na Argentina evidenciaram que a desigualdade de gênero, a escolaridade alcançada e a falta de conforto percebido no domicílio intensificam o sentimento de incerteza, medo e angústia e trazem a reflexão sobre o impacto ocasionado pela ruptura das relações afetivas e vínculos das pessoas durante o período de isolamento da COVID-19. Além disso, discutem a importância de se conhecerem os riscos para o surgimento de transtornos mentais e evidenciam a necessidade de atenção nas dimensões afetivas e de vínculos, que devem ser considerados no planejamento de políticas para o enfrentamento da COVID-19. O impacto sobre o convívio social apresentou aspecto relevante entre pessoas com maior escolaridade e renda (45,8\%). Entretanto, para pessoas de baixa renda e escolaridade, os problemas financeiros provocam maior impacto (35\%). Pessoas que residem em piores condiç̃̃es informaram disposição para permanecer menos tempo isoladas (73,9\%).

Em contrapartida, as medidas de isolamento devem ser transparentes e pautadas em evidências científicas, além de ser fundamental que sejam solidificadas por um bom diálogo entre os governantes e a população, atuando sobre as notícias negativas exibidas pela mídia sobre a COVID-19, além das fake news, que podem gerar, nos indivíduos, o estado de alerta constante, correlacionado ao medo de se contaminar e de morrer (Bezerra et al., 2020). Desse modo, esses indivíduos podem desenvolver transtorno de pânico que se caracteriza por crises intensas de ansiedade repentina com forte sensação de medo, acompanhadas de sintomas físicos, que podem ocorrer em qualquer lugar ou momento (Duarte et al., 2020). Os sentimentos de incerteza geram na população manifestações de sintomas de ansiedade, que podem evoluir para um quadro de depressão, caracterizando-se por uma mudança brusca no estilo de vida, levando à irritabilidade, a hábitos alimentares não saudáveis ou perda de apetite, a sentimentos de culpa e à perda de interesse para realizar atividades e passatempos que antes eram prazerosos para esses indivíduos (Wang et al., 2020; Zhang et al., 2020).

O estudo de Allen et al. (2020) se concentrou em um período de reabertura gradual, mas com taxas de infecção crescentes nos Estados Unidos. Uma porcentagem de 21\% dos participantes relataram sintomas depressivos, 16,58\% relataram sintomas elevados de ansiedade, e 5,38\%, níveis elevados de sintomas de trauma, tendo sido relatado também o aumento do uso indevido de álcool por 35,03\% dos participantes (35,59\% das mulheres e 33,08\% dos homens).

Os resultados dos estudos de Emuno et al. (2020) e Lu et al. (2020) apontam como necessária uma maior divulgação das práticas de preservação da saúde mental e da qualidade do sono recomendadas pelas associações de profissionais da saúde mental. Segundo os autores, é essencial a disponibilização de serviços on-line para atenção desses pacientes, sendo necessário trabalhar as questões relacionadas ao impacto do isolamento, e quais possíveis intervenções poderão ser adotadas. Apesar da representatividade dos dados relacionados à necessidade de se terem ferramentas para atuar no enfrentamento da crise de saúde mental ocasionado pela pandemia, eles não abrangem de forma direcionada como atuar agora, sendo recomendada cautela na interpretação e extrapolação de vários mecanismos apresentados como recurso para atender a essa demanda de cuidado.

Considerando o isolamento e o impacto dele sobre a saúde mental, identifica-se a necessidade de adotar medidas e planejamento que ultrapassem apenas a rede de cuidados especializados em saúde mental. O aporte dos profissionais das redes de atenção neste contexto de pandemia e pós-pandemia terá de ser presente e atuante no apoio emocional e psicológico. Também será importante planejar unidades de intervenção em crise em locais selecionados (como serviços de emergências) e em serviços de saúde mental em hospitais gerais para que possam acolher as demandas relacionadas à saúde mental da população (Roy et al., 2020; Emuno et al., 2020; Lu et al., 2020; Johnson et al., 2020).

\section{Conclusão}

A análise dos estudos incluídos nesta revisão evidencia que a população sente os efeitos do isolamento social diante da suspensão das atividades denominadas como essenciais, que envolvem as ocupações desempenhadas no âmbito escolar, no 
trabalho, na família, no lazer e no meio cultural. Assim, ao se deparar com essa realidade, a população teve de se adaptar para ressignificar as suas relações e dinâmicas sociais, afetivas e de trabalho.

Observa-se que os sintomas de alterações da saúde mental mais comumente ressaltados nos artigos são o estresse, o medo, a ansiedade, a culpa e a tristeza que geram sofrimento psíquico e podem ocasionar o surgimento de transtornos de pânico, transtornos de ansiedade, e depressão. Identificou-se também que o isolamento social e a diminuição de contato físico com as pessoas durante a pandemia não é, por si só, o único fator de risco para o adoecimento mental. Outros fatores foram identificados como influenciadores para o adoecimento, tais como: a renda familiar diminuída em razão dos impactos da doença na economia mundial, a exposição às informações relacionadas à doença COVID-19 (número de pessoas contaminadas, mortes, sistema de saúde colapsado).

E, por fim, os resultados encontrados neste estudo enfatizam a importância do acolhimento e da terapia, especificamente na modalidade de atendimento on-line, fornecendo orientações em saúde, para atender às necessidades da comunidade, especialmente os com maior risco de desenvolver algum transtorno mental. Essas medidas visam a fortalecer as relações afetivas com rodas de conversas virtuais ou acolhida virtual. Entretanto, faz-se necessário fortalecer também os serviços de saúde para a demanda desses atendimentos no período pós-pandêmico, por meio de medidas que possibilitem ao indivíduo ter esperança no contexto futuro de saúde, reduzindo consideravelmente as consequências do isolamento social na saúde mental. Importante ressaltar e refletir que, embora a pandemia imponha desafios adicionais à saúde mundial, ela também colabora com o aprimoramento de práticas e da pesquisa em condições de crise, emergência e desastre.

\section{Referências}

Alomo, M., Gagliardi, G., Peloche, S., Somers, E., Alzina, P., \& Prokopez, C. R. Efectos psicológicos de la pandemia COVID-19 en la población general de Argentina [Psychological effects during the COVID-19 outbreak in Argentina]. Rev Fac Cien Med Univ Nac Cordoba. 25;77(3):176-181. 10.31053/1853.0605

Allen, C. S., et al. (2020) Mental health outcomes associated with the COVID-19 pandemic: Prevalence and risk factors in a southern US state, Psychiatry Research, 293, 113476, 10.1016/j.psychres.2020.113476

Andrés, L. B., et al. (2020). Diferencias en función de la edad y la autopercepción del envejecimiento en ansiedad, tristeza, soledad y sintomatología comórbida ansioso-depresiva durante el confinamiento por la COVID-19, Revista Española de Geriatría y Gerontología, 55(5), 272-278. 10.1016/j.regg.2020.05.005

Ahmed, M. Z., Ahmed, O., Aibao, Z., Hanbin, S., Siyu, L., \& Ahmad, A. Epidemic of COVID-19 in China and associated Psychological Problems. Asian J Psychiatr. 10.1016/j.ajp.2020.102092

Atchimson, C., Bowman, L., Vrinten, C., Redd, R., Pristera, P., Eaton, J. W., \& Ward, H. (2020). Perceptions and behavioural responses of the general public during the COVID-19 pandemic: A cross-sectional survey of UK Adults. medRxiv 2020; 04.01.20050039. 10.1590/1413-81232020256.1.10472020

Barros, M. B. A., et al. (2020). Relato de tristeza/depressão, nervosismo/ansiedade e problemas de sono na população adulta brasileira durante a pandemia de COVID-19. Epidemiologia e Serviços de Saúde, 29(4), e2020427. 24, 10.1590/s1679-49742020000400018

Bezerra, A. C. V., Silva, C. E. M. D., Soares, F. R. G., \& Silva, J. A. M. D. (2020). Fatores associados ao comportamento da população durante o isolamento social na pandemia de COVID-19. Ciência \& Saúde Coletiva, 25, 2411-2421. 10.1590/1413-81232020256.1.10792020

Brooks, S. K., Webster, R. K., Smith, L. E., Woodland, L., Wessely, S., Greenberg, N., \& Rubin, G. J. (2020). The psychological impact of quarantine and how to reduce it: rapid review of the evidence. The Lancet, 395(102227), 912-920. 10.1016/S0140-6736(20)30460-8

Chakraborty, K., \& Chatterjee, M. (2020). Psychological impact of COVID-19 pandemic on general population in West Bengal: A cross-sectional study. Indian journal of psychiatry, 62(3), 266-27210.4103/psychiatry.IndianJPsychiatry_276_20

Delmastro M, \& Zamariola, G. (2020). Depressive symptoms in response to COVID-19 and lockdown: a cross-sectional study on the Italian population. Scientific reports, 10(1), 22457. 10.1038/s41598-020-79850-6

Duarte, M. D. Q., Santos, M. A. D., Lima, C., Giordani, J. P., \& Trentini, C. M. (2020). COVID-19 e os impactos na saúde mental: uma amostra do Rio Grande do Sul, Brasil. Ciência \& Saúde Coletiva, 25, 3401-3411. 10.1590/1413-81232020259.16472020

Enumo, S. R. F., Weide, J. N., Vicentini, E. C. C., Araujo, M. M., \& Machado, W. L. (2020). Enfrentando o estresse em tempos de pandemia: proposição de uma Cartilha. Estudos de Psicologia (Campinas), 37, e200065. 10.1590/1982-0275202037e200065

Johnson, M. C., Saletti-Cuesta, L., \& Tumas, N. (2020). Emociones, preocupaciones y reflexiones frente a la pandemia del COVID-19 en Argentina. Ciência \& Saúde Coletiva, 25(Suppl. 1), 2447-2456. 10.1590/1413-81232020256.1.10472020 
Hortulanus, R., Machielse M., \& Meeuwesen, L. (2006). Social isolation in modern society. Routledge.

Holmes, E. A., O'Connor, R. C., Perry, V. H., Tracey, I., Wessely, S., Arseneault, L., Ballard, C., Christensen, H., Cohen Silver, R., Everall, I., Ford, T., John, A., Kabir, T., King, K., Madan, I., Michie, S., Przybylski, A. K., Shafran, R., Sweeney, A., Worthman, C. M., Yardley, L., Cowan, K., Cope, C., Hotopf, M., \& Bullmore, E. Multidisciplinary research priorities for the COVID-19 pandemic: a call for action for mental health science. Lancet Psychiatry. 7(6):547-560. $10.1016 /$ S2215-0366(20)30168-1

Lana, R. M., et al. (2020). Emergência do novo coronavírus (SARS-CoV-2) e o papel de uma vigilância nacional em saúde oportuna e efetiva. Cadernos de Saúde Pública, 36(3), e00019620. 10.1590/0102-311x00019620

Lu, P., Li, X., Lu, L., \& Zhang, Y. (2020) The psychological states of people after Wuhan eased the lockdown. PLoS ONE 15(11): e0241173. 10.1371/journal.pone.0241173

Mendes, K. D. S., et al. (2008). Revisão integrativa: método de pesquisa para a incorporação de evidências na saúde e na enfermagem. Texto \& Contexto Enfermagem, 17(4), 758-764. 10.1590/S0104-07072008000400018

Moher, D., et al. The PRISMA Group (2009). Preferred Reporting Items for Systematic Reviews and Meta-Analyses: The PRISMA Statement. J Clin Epidemiol. 10.1016/j.jclinepi.2009.06.005

OMS. Coronavirus disease (COVID-19) pandemic. 2020c. https://www.who.int/emergencies/diseases/novel-coronavirus-2019.

Ozamiz-Etxebarria, N., et al. (2020). Niveles de estrés, ansiedad y depresión en la primera fase del brote del COVID-19 en una muestra recogida en el norte de España. Cadernos de Saúde Pública, 36(4), e00054020. 10.1590/0102-311x00054020

Ramírez-Ortiz, J., Castro-Quintero, D., Lerma-Córdoba, C., Yela-Ceballos, F., \& Escobar-Córdoba, F. (2020). Consecuencias de la pandemia COVID-19 en la Salud Mental asociadas al aislamiento social. SciELO Preprints, 1, 1-21. 10.1590/SCIELOPREPRINTS.303

Roy, D., Tripathy, S., Kar, S. K., Sharma, N., Verma, S. K., \& Kaushal, V. (2020). Study of knowledge, attitude, anxiety \& perceived mental healthcare need in Indian population during COVID-19 pandemic. Asian J. Psychiatry, 51(1), 1-16..1016/j.ajp.2020.102083

Rezende, J. M. (2009). À sombra do plátano: crônicas de história da medicina [online]. São Paulo: Editora Unifesp, 2009. As grandes epidemias da história. pp. 73-82. http://books.scielo.org/id/8kf92/pdf/rezende-9788561673635-08.pdf

Santos, C. M. C., et al. (2007). A estratégia PICO para a construção da pergunta de pesquisa e busca de evidências. Revista Latino-Americana de Enfermagem, 15(3), 508-511. 10.1590/S0104-11692007000300023

Shigemura, J., et al. (2020). Public responses to the novel 2019 coronavirus (2019-nCoV) in Japan: mental health consequences and target populations. Psychiatry clin. neurosci.; 4(4):281-2. https://www.ncbi.nlm.nih.gov/pmc/a rticles/PMC7168047/pdf/PCN-74- 281.pdf

Wang, C., et al. (2020). A longitudinal study on the mental health of general population during the COVID-19 epidemic in China. Brain Behav Immun. 10.1016/j.bbi.2020.04.028

Wang, C., et al. (2020). Immediate Psychological Responses and Associated Factors during the Initial Stage of the 2019 Coronavirus Disease (COVID-19) Epidemic among the General Population in China. Int J Environ Res Public Health. 17(5):1729. 10.3390/ijerph17051729

World Health Organization. (2020). Getting your workplace ready for COVID-19, Genebra. https://www.who.int/docs/default-source/coronaviruse/gettingworkplace-ready-for-covid-19.pdf?sfvrsn=359a81e7_61

Xiao, C. (2020). A novel approach of consultation on 2019 novel coronavirus (COVID-19)-related psychological and mental problems: structured letter therapy. Psychiatry Investigation, 17(2), 175-176. 10.30773/pi.2020.0047

Zhang, J., Lu, H., Zeng, H., Zhang, S., Du, Q., Jiang, T., \& Du, B. (2020). The differential psychological distress of populations affected by the COVID-19 pandemic. Brain, behavior, and immunity, 87, 49-50. 10.1016/j.bbi.2020.04.031 